Analytica Chimica Acta, 81 (1976) $21-30$

o Elsevier Scientific Publishing Company, Amsterdam - Printed in The Netherlands

\title{
'THE DEVELOPMENT OF A FULLY COMPUTERIZED SYSTEM FOR SAMPLED D.C. POLAROGRAPHY WITH STANDARD INTERFACING
}

\author{
M. BOS \\ Department of Chemical Technology. Twente University of Technology. Fnschede \\ (The Netherlonds)
}

(Received 4 th June 1975)

SUMMARY

A complete system, based on the online PDP-11 computer (Digital Equipment Corporation) was developed for computerized sampled d.c. polarography with direct digital control. The system includes compensation of ohmic cell resistance and processing of the polarographic data. The accuracy of the system in the determination of the various polarographic parameters is: diffusion current $\pm 2 \%$, half-wave potential $: 2 \mathrm{mV}$, and slope of the $\log$ plot $=2 \mathrm{mV}$.

Process computers have been used to control [1,2] and evaluate [3] stationary electrode polarography, and the idea of programming a digital online computer to perform sampled d.c.-polarography is not new $[4]$. However, no reports about this subject have appeared in the literature, and important questions with regard to the accuracy and sensitivity that can be ohtained have remained unanswered. This investigation was started to answer these questions, and to evaluate different methods of processing polarographic data with respect to speed, accuracy and the amount of information extracted from the data. Multiparametric curve-fitting methods were chosen for the data processing, as these methods are also suitable for polarographic waves which have the diffusion plateau obscured, a rather common situation in polarographic analysis which greatly limits the possibilities of conventional polarography. Finally this study was also undertaken to obtain a rough estimate of the total effort in the development of a fully computerized analytical method.

EXPERIMENTAI.

\section{Chemicals}

Cadmium chloride (Analar), potassium chloride (Merck, reagent grade) and mercury (DRIJFHOU'T, Polarographic grade) were used as received. Tetraethylammonium perchlorate (TEAP; Eastman) was recrystallized from ethanol (Merck, reagent grade) and dried in vacuo at $40^{\circ} \mathrm{C}$. 


\section{Apparatus}

A Radiometer PO4 polarograph, equipped with a Radiometer DLT1 droplife timer, was used in the experiment for comparison purposes; it was adapted to three-electrode operation with a Sargent model $A i R$ compensator. The characteristics of the dropping mercury electrode used were (at open circuit) in $1 \mathrm{M} \mathrm{KCl}: m=3.98 \mathrm{mg} \mathrm{s}^{-1} t=2.39 \mathrm{~s}$. In the double-walled thermostatted $\left(20.00 \pm 0.05^{\circ} \mathrm{C}\right)$ polarographic cell, a saturated calomel reference electrode was used with a mercury pool at the bottom of the vessel as auxiliary electrode.

The "computer polarograph" consisted of the Radiometer droplife timer and a digital online computer (PDP11/20, Digital Equipment Corporation) with the following standard accessories: 1 : : type ASR 33; Dectape unit TU 56; Analog to Digital conversion subsystem ADO1 ( 2 channels in use), resolution 10 bits $+\operatorname{sign}$, ranges $\pm 2.50 \mathrm{~V}, \pm 5.00 \mathrm{~V}, \pm 10.00 \mathrm{~V}$; Digital to Analog conversion subsystem $A$ A-11-D ( 4 channels in use), resolution 11 bits + sign, range $\pm 10 \mathrm{~V}, 10 \mathrm{~mA}$

The memory size was $8 \mathrm{~K}$ words ( $16 \mathrm{bits}$ ). 'The D/A conversion subsystem AA-11-D can provide $\pm 10 \mathrm{~V}$ at $10 \mathrm{~mA}$, so it is possible to drive the polarographic cell directly from this source. Furthermore a $+10 \mathrm{~V}$ signal from this $D / A$ converter can also directly drive the synchronization input of the droplife timer.

The polarographic cell used with this equipment was of the same type as that used for the Radiometer PO4 experiments. The characteristics of the dropping mercury electrode used with this "computer polarograph" were as follows: $m-1.29 \mathrm{mg} \mathrm{s}^{-1}, t=5.40 \mathrm{~s}$ in $1 \mathrm{M} \mathrm{KCl}$, and $m: 1.30 \mathrm{mg} \mathrm{s}^{-1}$, $t=5.72 \mathrm{~s}$ in $0.1 \mathrm{M}$ TEAP- $50 \%$ ethanol. A saturated calomel electrode or a silver/silver chloride electrode of the double junction type (Ingold 373-90NS-M5) served as reference electrode; the inner compartment of the latter was filled with saturated potassium chloride, while the outer compartment was filled with $0.1 \mathrm{M}$ TEAP in $50 \%$ ethanol. A diagram of the complete equipment for computerized sampled d.c. polarography is given in Fig. 1.

\section{Polarographic procedure}

The sample was placed in the cell, with mercury as auxiliary electrode. The solution was decxygenated for $15 \mathrm{~min}$ with nitrogen purified by passing it over copper heated to $350^{\circ} \mathrm{C}$. The polarographic curve was then recorded. Cadmium samples in $1 \mathrm{M}$ potassium chloride as supporting electrolyte were prepared from a stock solution of cadmium chloride standardized against EDTA with xylenol orange as indicator. Potassium samples in 0.1 M TEAP supporting electrolyte were prepared from a stock solution made from a weighed amount of dried potassium chloride. 


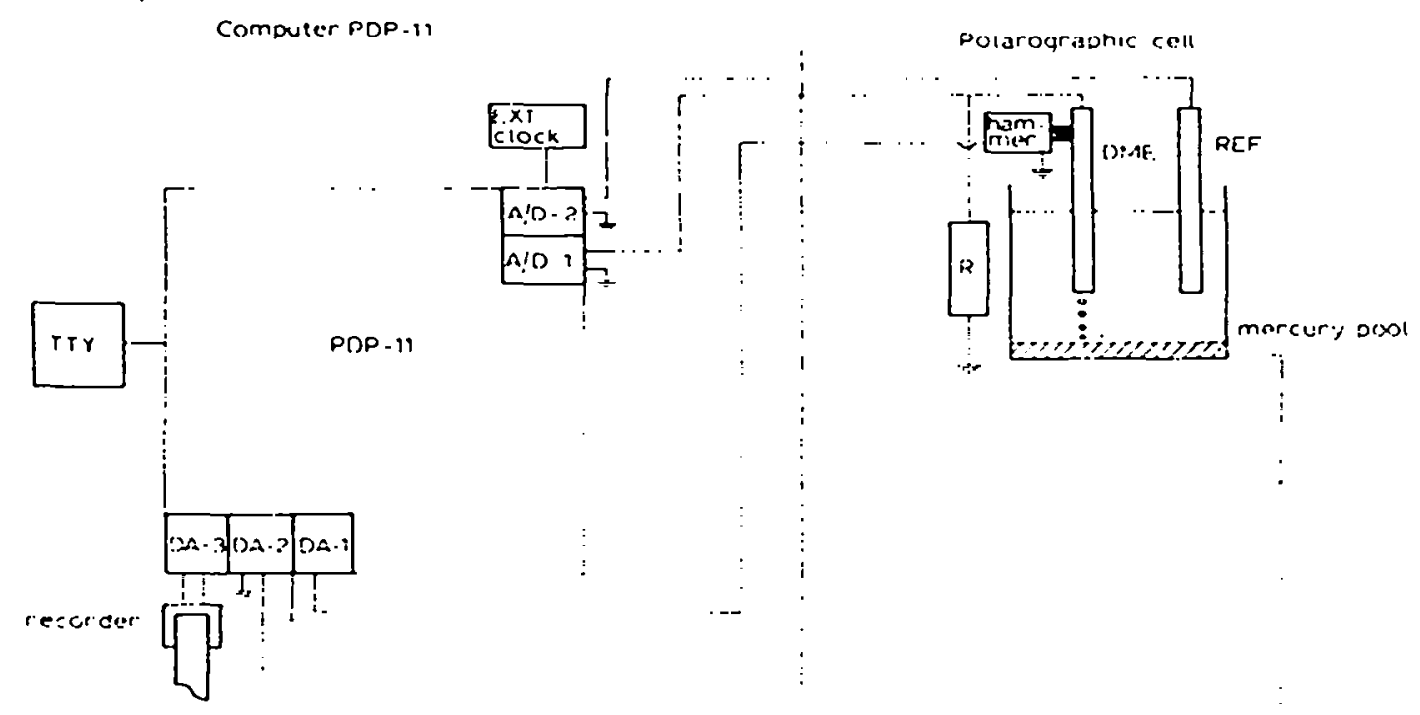

Fig. 1. The "cumputer polarograph".

\section{Computer programs}

\section{Program for digital control of polarography}

In carrying out the polarographic experiment. the computer has to perform the following functions:

1. maintain the potential of the dropping mer(ury electrode (I)MIF) at a set point value versus the reference electrode by changing the potential of the auxiliary electrode (mercury pool);

2. change this set point value of the potential of the DME linearly with time;

3. measure and store the cell current with a relative accuracy 0.1 ; of it:s full scale value;

1. synchronize the measurement of cell current with the mercury drop life: and

5. dislodge the mercury drop at fixed intervals.

Furthermore, in order to obtain the optimal conditions. the oprerator must be able to choose the drop life time. the potential from which the scain starts, the direction of the scan (i.e. cathodic or anodic), the potential at which the scan stops, the scan rate, the sensitivity of the current measurement. and the amplification in the ohmic cell resistance compensation circuit.

For the convenience of the operator, it is also desirable to have the following facilities: (a) a display of the recorded polarogram on an 
oscilloscope; (b) the possibility of obtaining a hard copy of the polarogram on a chart recorder; and (c) the possibility of repeated recording of the polarographic curve for the same sample.

A flow chart of the computer program that meets all these requirements is given in Fig. 2. The symbols used for the various parameters in the program are listed in Table 1.

'The PDP-11/20 online computer features interrupt facilities at different priorities. Real-time operations are programmed with:the use of these interrupt facilities for a line frequency clock and separate external clock for the $\mathrm{A} / \mathrm{D}$ conversion. At the right moments these clocks cause an interrupt, i.e. they interrupt the normal flow in program execution, start the execution of a specific service routine, and when this service routine has been completed, resume normal program execution.

The program starts with a conversational part in which the parameters set by the operator are input to the computer via the teletype. 'Then a waitloop follows where the computer waits for the start command. After the start command has been received, the values of SCANPO and CURREN are set. Then the line clock $(50 \mathrm{~Hz})$ is started and the $A / D$ converter is activated. From this point every time that a pulse of the external clock is encountered, the $A / D$ converter measures the potential between the DMF and the reference electrode. With these values the adjustment of the potential of the auxiliary electrode is calculated: PROPOR $\times$ (SCANPO - POIDME + POTREF). The calculated adjustment is applied to I) A channel 2 . If more?

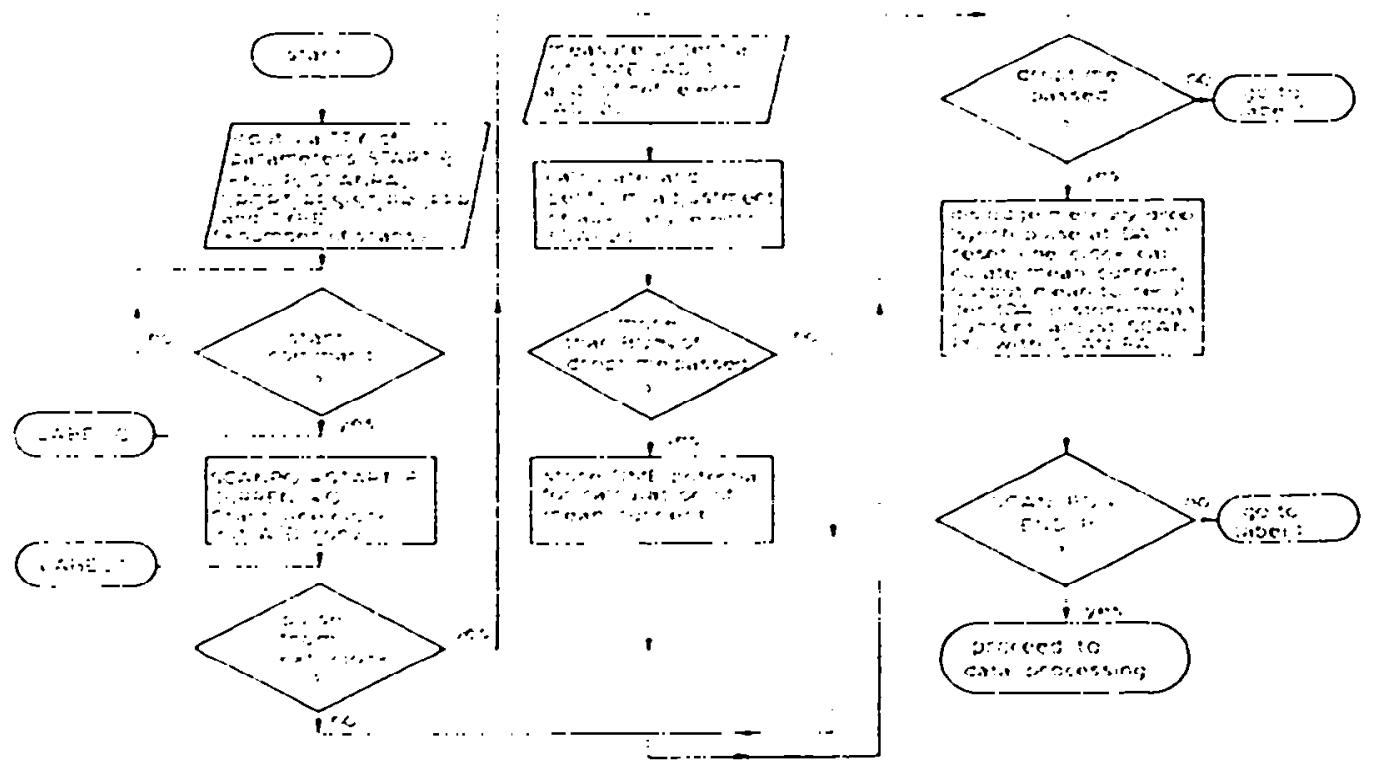

Fig. 2. Flow diagram of program for digital control of polarography. 
TABLE 1

Symbols used in flow chart of computer programs

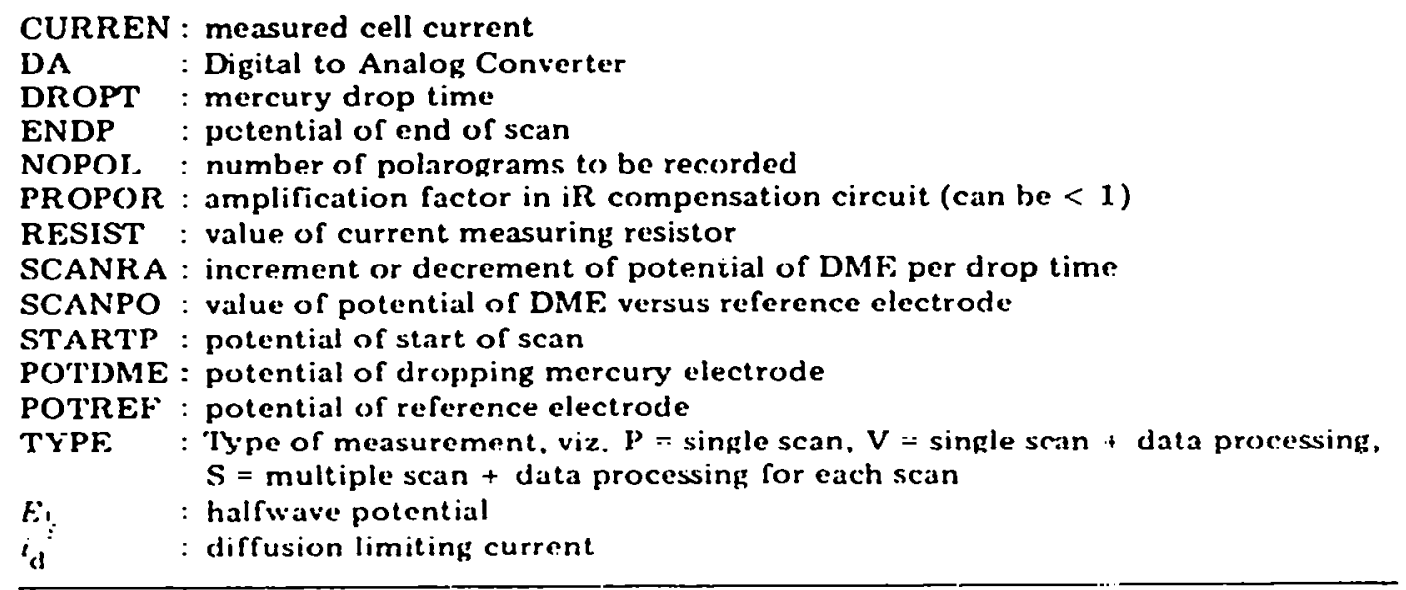

than $80 \%$ of the drop time has elapsed, the value POTDME is stored for later calculation of the mean current during the last $20 \%$ of drop life. I ine clock pulses are counted to determine the stage of the drop life. Ithen the drop time is complete the mercury drop is dislodged by a synchronization pulse of DA-1. The line clock counter is reset and the mean value of the current during the last $20 \%$ of drop life is calculated. This value of the mean current is output to a chart recorder at DA-3, and is also stored in the computer memory. Then the value of SCANPO is adjusted with SCANIRA. Finally a check is made that the end of the scan has been reached. If this test is successful, the program proceeds to the data-processing part; if not, the computer must wait for a new pulse of the external clock.

Program for processing polarographic data

Especially when polarography is used not only for analytical purposes, but also to elucidate electrode processes, the handling of the polarographic data should include calculation of the half-wave potential and the slope of the log plot, as well as calculation of the limiting current, and digital filtering of the signal to reduce noise. A simple but effective way of filtering the polarographic: signal digitally is the method given by Savitzky and Golay [5].

If the theoretical equation describing the experiment is known, curvefitting methods can provide the values of the parameters which determine? the result. Most polarographic waves are described by the equation [6]

$E=E_{!}-\frac{R T}{\alpha n F} \ln \left(\frac{i}{i_{\mathrm{d}}-i}\right)$

which can be rewritten as [7] 
$i=i_{\mathrm{d}} /\left\{\exp \left[\left(E-E_{\mathfrak{i}}\right) / S\right]+1\right\}$

where $S=R T / \alpha n F$.

It should be noted that the program which utilizes this equation can be applied only to the polarography of substances which have a reduction product that is either soluble in mercury or in the medium.

For curve fitting based on eqn. (2), the rigorous least-squares method given by Wentworth 18] for three parameters was chosen, mainly because of its computational speed; this method requires reasonable initial estimates of the parameters wanted. The initial estimate of the diffusion current can be found from the difference in the currents at two poinis, one before and one after the rising part of the polarcgraphic wave. An initial estimate of the half-wave potential of a polarographic wave can easily be found from the zero crossing point in the second derivative of the polarographic curve. Equation (2) applies only to the points of the rising part of the polarographic wave, so that it is necessary to determine which points belong to that part. This can be accomplished by means of the first derivative of the polarographic: curve: when this first derivative exceeds a certain threshold value the corresponding points in the polarographic curve are on the rising part of a wave. The first and second derivatives of the polarographic curve can be calculated by a procedure similar to that used for the smoothing [5]. A flow chart of the complete program for the processing of the polarographic data is given in Fig. 3. The program operates on data stored in the computer memory by the program for digital control of polarography described in the previous section. Throughout the program, standard computational DEC subroutines are used.

\section{RESIJITS}

The programs were written in DEC PAL-11 A assembly language. The total memory size needed to aceommodate the complete set of programs is about $15 \mathrm{~K}$ words (16 bits) including the memory space reserved for storage of the data. If only $8 \mathrm{~K}$ memory is available, a magnetic tape unit can be used to store the complete set of programs, and the appropriate programs are brought into the memory as required. Data-processing programs are not required while the experiment is running, whereas the digital-control program can be removed from the memory during data processing. Development of programs, debugging and experimental testing of the complete system took about 600 man-hours, of which 300 were spent at the computer. The program that controls the experiment has the following features:

1. the potential at which the scan starts and ends can be set to any integral value in the range $\pm 5000 \mathrm{mV}$;

2 . the scan rate can be adjusted in $1-\mathrm{mV}$ steps down to $\pm 1 \mathrm{mV}$ per drop time;

3. the lowest drop time is $1 \mathrm{~s}$, and this can be increased by 1 -s increments;

4 . the cell current is measured during the last $20 \%$ of the drop life; 


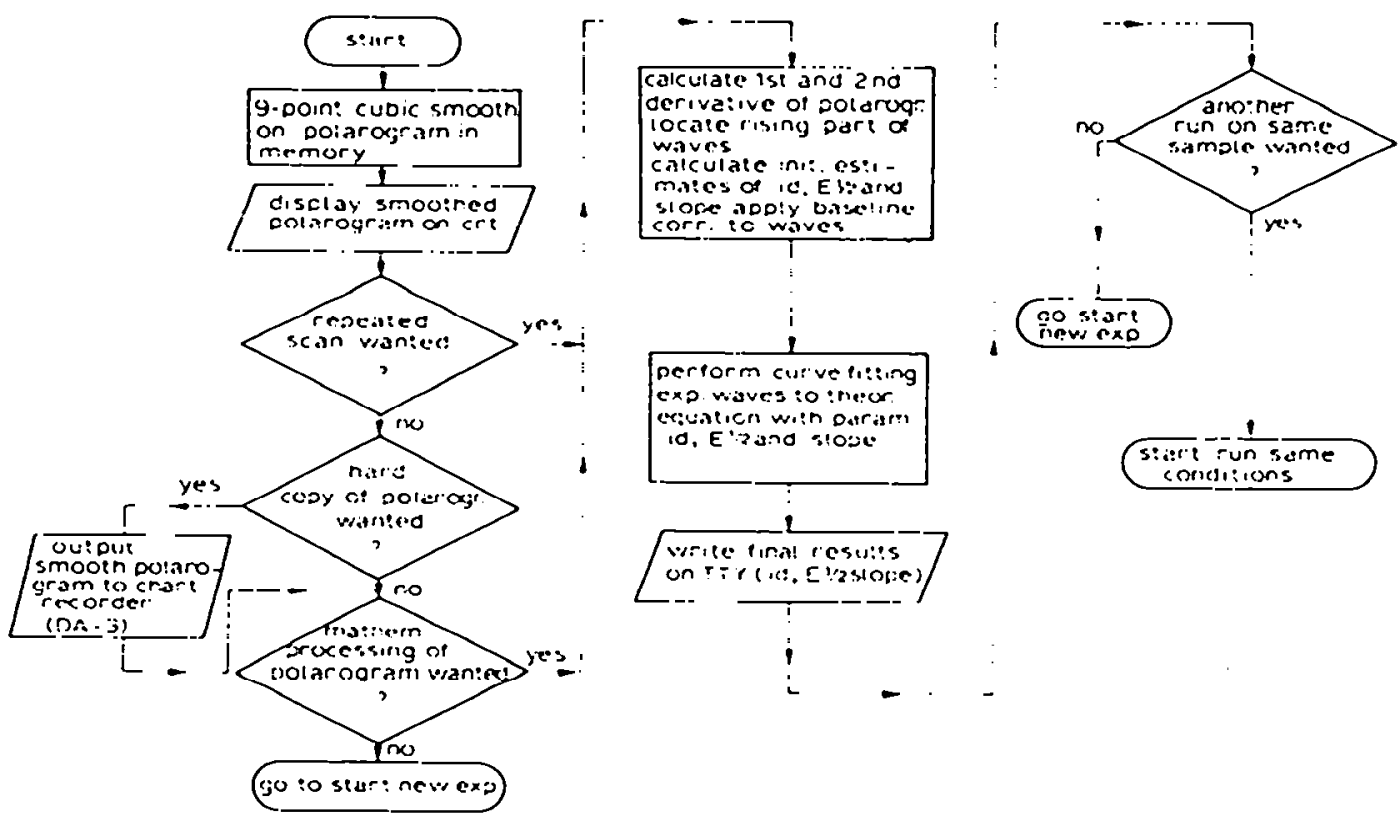

Fig. 3. Flow chart of program for processing polarographic data.

5. the ramp voltage increment (scan rate) is synchronized with the drop fall;

6 . the polarographic curve can be recorded (strip chart) during the experiment;

7. the smoothed polarographic curve can be shown on the chart recorder after display on an oscilloscope;

8. three-electrode operation provides for iR-compensation;

9. repeated scans and data processing on the same sample are possible without operator intervention.

The sensitivity of the current measurement is not under computer control, but can be changed by changing the measuring resistance $R$ (Fig. 1 ).

'The data-processing program produces for each wave in the polarographic curve: (a) current versus voltage values for all points on the rising part of the wave; and (b) values for the limiting current, half-wave potential and slope of the log plot. Baseline correction for the waves is done by extrapolating a least-squares line drawn through 20 points, starting 10 points before the polarographic wave, from the starting potential side. For anodic scans, calculations are adjusted appropriately.

Initially, the brute-force multiparametric curve-fitting method of Meites [7] was used in the calculations, but for waves with about 30 points the computer time needed exceeds $20 \mathrm{~min}$. With the Wentworth method [8] computer time for the processing of one polarographic wave with 30 
points, is about $1 \mathrm{~min}$ and proportionately less for waves with fewer points (higher scan rate).

The results for the reduction of cadmium in $1 \mathrm{M}$ potassium chloride as supporting electrolyte by computerized sampled d.c. polarography are given in Table 2. The experimental conditions were: starting potential, $-0.300 \mathrm{~V}$; final potential, $-0.750 \mathrm{~V}$; scan rate $0.004 \mathrm{~V} / 2 \mathrm{~s}$; drop time, $2 \mathrm{~s}$; measuring resistance value, $80.000 \Omega$. For comparison, Table 2 also shows the results of similar experiments run on a commercial polarograph; in this case the $E_{f}$ values and diffusion currents were determined gxaphically on the recorder paper.

Figure 4 shows the polarographic wave recorded by the "computer polarograph" for the reduction of potassium ion in $0.1 \mathrm{M}$ tetraethylammonium perchlorate in $50 \%$ ethanol as supporting electrolyte. The conditions were: starting potential, $-1.500 \mathrm{~V}$; final potential, $-2.052 \mathrm{~V}$; scan rate, $0.006 \mathrm{~V} \mathrm{~s}^{-1}$; drop time, $1 \mathrm{~s}$; measuring resistance, $80.000 \Omega$. No diffusion current plateau

TABILF, 2

Sampled d.c. polarography of cadmium(II) in $1 \mathrm{M} \mathrm{KCl}$ by the computerized method and by the Radiometer POA method

\begin{tabular}{|c|c|c|c|c|c|c|c|}
\hline \multirow{2}{*}{$\begin{array}{l}\text { Concen. } \\
\left(-10^{+} \mathrm{M}\right)\end{array}$} & \multicolumn{4}{|c|}{ Computerized method } & \multicolumn{3}{|c|}{ POI method (2-s drop time) } \\
\hline & $i_{(\mu \mathrm{d})}$ & $\begin{array}{l}E_{t} \\
(\dot{v})\end{array}$ & $\begin{array}{l}\text { Slope } \\
(m V)\end{array}$ & $l_{d}^{a}$ & $i_{(\mu \mathrm{d})}$ & $\begin{array}{l}E_{!} \\
(\dot{V})\end{array}$ & $I_{\mathrm{d}^{2}}$ \\
\hline 2.026 & 1.183 & -0.6440 & 27.55 & 3.81 & 2.80 & -0.635 & 4.27 \\
\hline $2.026 i$ & 1.206 & -0.6440 & 29.96 & 3.89 & 2.72 & -0.640 & 4.14 \\
\hline 2.026 & 1.215 & -0.6444 & 29.27 & 3.91 & 2.66 & -0.635 & 1.05 \\
\hline 3.039 & 1.766 & $-0.6+36$ & 28.61 & 3.79 & 3.96 & -0.640 & 4.02 \\
\hline 3.039 & 1.790 & -0.6443 & 28.95 & 3.84 & 4.00 & -0.640 & 4.06 \\
\hline 3.039 & 1.761 & -0.6444 & 28.54 & 3.78 & - & -- & - \\
\hline 4.052 & 2.452 & -0.6427 & 29.53 & 3.95 & 5.30 & -0.635 & 4.04 \\
\hline 4.052 & 2.410 & -0.6429 & 29.57 & 3.88 & 5.20 & -0.635 & 3.96 \\
\hline 4.052 & 2.418 & -0.6434 & 28.98 & 3.90 & -- & - & - \\
\hline 5.065 & 2.984 & $\cdots 0.6445$ & 28.15 & 3.85 & 6.64 & -0.640 & 4.05 \\
\hline 5.065 & 3.029 & -0.6449 & 29.64 & 3.90 & - & - & - \\
\hline 5.065 & 2.989 & -0.6437 & 29.04 & 3.85 & - & - & - \\
\hline 6.078 & 3.654 & -0.6450 & 29.11 & 3.92 & 8.00 & -0.645 & 4.06 \\
\hline 6.078 & 3.602 & -0.6449 & 28.49 & 3.87 & 7.94 & -0.640 & 4.03 \\
\hline 6.078 & 3.590 & -0.6448 & 29.39 & 3.86 & 7.92 & -0.635 & 4.02 \\
\hline 8.10 .1 & 4.796 & -0.6417 & 28.93 & 3.86 & 10.56 & -0.640 & 4.02 \\
\hline 5.104 & 4.825 & -0.6414 & 29.60 & 3.89 & 10.32 & -0.640 & 3.93 \\
\hline 8.104 & $4.80 \pi$ & -0.6417 & 29.04 & 3.92 & 10.16 & -0.640 & 3.87 \\
\hline 10.130 & 5.990 & -0.6422 & 29.14 & 3.86 & 13.20 & -0.640 & 4.02 \\
\hline 10.130 & 5.960 & -0.6420 & 28.75 & 3.84 & 12.94 & -0.635 & 3.94 \\
\hline 10.130 & 6.014 & -0.6419 & 29.48 & 3.88 & 13.22 & -0.635 & 1.06 \\
\hline Mean & & -0.643 & 29.0 & 3.87 & & -0.638 & 4.03 \\
\hline$s$ & & 0.001 & 0.5 & 0.04 & & 0.003 & 0.09 \\
\hline
\end{tabular}

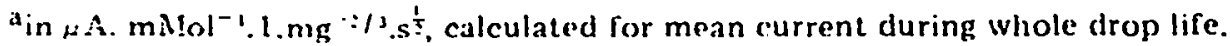




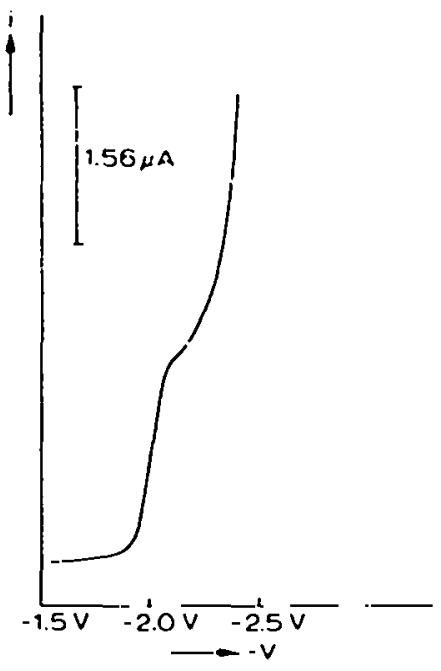

Fig. 4. Sampled d.c. polarogram for $8 \cdot 10^{-} \mathrm{M} \mathrm{K} \cdot$ in $0.1 \mathrm{M} \mathrm{TE} \mathrm{AP}-50$ \% ethanol.

TABLE 3

Computerized sampled d.c. polarography of potassium(I) in $0.1 \mathrm{M}$ TEAP in $50 \%$ ethanol, with 1-s drop time

\begin{tabular}{lllll}
\hline $\begin{array}{l}\text { Concn } \\
\left(10^{-4} \mathrm{M}\right)\end{array}$ & $\begin{array}{l}i_{\mathrm{d}} \\
(\mu \mathrm{A})\end{array}$ & $\begin{array}{l}E_{\frac{1}{2}}{ }^{\mathrm{V}} \\
(\mathrm{V})\end{array}$ & $\begin{array}{l}\text { Slope } \\
(\mathrm{mV})\end{array}$ & $i_{\mathrm{d}}^{\mathrm{b}}$ \\
\hline 3.00 & 0.769 & -1.9574 & 58.7 & 1.87 \\
3.00 & 0.760 & -1.9573 & 55.2 & 1.84 \\
4.00 & 1.005 & -1.9603 & 56.7 & 1.83 \\
4.00 & 1.060 & -1.9625 & 61.6 & 1.93 \\
4.00 & 1.009 & -1.9598 & 57.5 & 1.8 .4 \\
4.00 & 1.030 & -1.9621 & 58.7 & 1.79 \\
5.00 & 1.231 & -1.9629 & 59.2 & 1.81 \\
5.00 & 1.245 & -1.9615 & 58.9 & 1.80 \\
5.00 & 1.240 & -1.9618 & 59.0 & 1.83 \\
5.00 & 1.254 & -1.9619 & 59.1 & 1.84 \\
5.00 & 1.264 & -1.9615 & 60.0 & 1.88 \\
6.00 & 1.547 & -1.9640 & 60.7 & 1.89 \\
6.00 & 1556 & -1.9652 & 61.9 & 1.87 \\
6.00 & 1.538 & -1.9643 & 59.3 & 1.80 \\
8.00 & 1.982 & -1.9639 & 61.1 & 1.84 \\
8.00 & 1.979 & -1.9653 & 60.9 & 0.0 .1 \\
Mean & & -1.962 & 59.3 & 1.8 \\
$s$ & & 0.002 & & \\
\hline
\end{tabular}

a Versus $\mathrm{Ag} / \mathrm{Ag} \mathrm{Cl}$ (salurated $\mathrm{KCl}$ ).

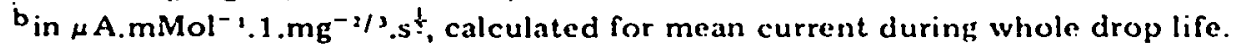


can be observed, but the data-processing program is still successful as indicated by the results in Table 3.

\section{CONCLUSIONS}

The results prove that even in unfavourable cases such as the determination of potassium, the accuracy of the computerized sampled d.c. polarography is $\pm 2 \%$ for the diffusion current constant, $\pm 2 \mathrm{mV}$ for the half-wave potential and $+2 \mathrm{mV}$ for the slope of the log plot. These errors compare favourably with the conventional method. Moreover, considerable time can be saved by processing the experimental data by computer. Diffusion current, half-wave potential and slope of the log plot are obtained within $1 \mathrm{~min}$ of finishing the experiment.

The use of Assembly language in the development of the programs for the data processing proved to be uneconomical. About one half of the development time was used in writing and debugging these programs. Partly this can be accounted for as follows: Initially a Basic program written by Meites [7] was used for the multiparametric curve-fitting, but execution of this program on the PDP-11 was very slow ( $>60$ min for waves with 30 points); the use of assembly language reduced the time considerably, but not enough to be of real practical value. Only with the Wentworth approach [8] programmed in assembly language, could data-processing times of $1 \mathrm{~min}$ be achicved.

It is estimated that the total effort for the development of the computerized sampled d.c. polarography system could have been reduced by $40 \%$ by the use of fortran in the data-processing programs. 'The use of assembly language is only recommended where short execution times are mandatory.

'The author wishes to thank Ir. II. J. de Groot for his great help with the computer programs, Prof. E. A. M. F. Dahmen for his support, and Mrs. B. Verbeeten-v. Hettema for preparing the manuscript.

\section{REFERENCES}

1 S. P. Pcrone, J. E. Harrar, F. B. Stephens and R. E. Anderson, Anal. Chem., 40 (1968) 899.

2 S. P. Perone, D. O. Jones and W. F. Gutknecht, Anal. Chem., 41 (1969) 1154.

3 W. F. Gutknecht and S. P. Perone, Anal. Chem., 42 (1970) 906.

4 D. E. Smith, in J. S. Mattson, H. B. Mark and H. C. MacDonald (eds), Electrochemistry, Vol. 2, Marcel Dekker, New York, 1972, p. 416.

5 A. Savitzky and M. J. E. Golay. Anal. Chem., 36 (1964) 1627.

6 J. Ieyrovský and J. Kuta, Grundlagen der Polarographie. Akademie Verlag, Berlin, 1965. p. 99.

7 T. Meites and L. Meites, Talanta, 19 (1972) 1131.

6 W. F. Wentworth, J. Chem. Educ., 42 (1965) 96. 